\title{
ANALYTICAL AND NUMERICAL STUDY OF SOME VARIANTS OF KOITER'S LINEAR MODEL OF THIN SHELLS ${ }^{1}$
}

\author{
Ángeles Vilariño Moreno
}

\begin{abstract}
Koiter's linear model for thin shells is obtained from the classic equations of the three-dimensional linear elasticity with the Kirchhoff-Love hypothesis; the variety of formulations of this model is based on the precision of the analysis carried out. In this work we detail these simplifications, and analyse the origin and the error of some variations of the model. We also approximate some of these versions by a nonconforming finite element method and compare the numerical results over some classical bench-marks.
\end{abstract}

\section{Introduction}

A thin elastic shell is a three-dimensional elastic solid of which one dimension is small with respect to the two others; this particular geometry leads to two-dimensional models formulated in terms of the middle surface of the shell to study its deformation. Different authors have deduced different shell models from the classic theory of the three-dimensional elasticity with the Kirchhoff-Love hypothesis; this variety of equations is based on different interpretations of the cited hypothesis, on a ledger or smaller degree of precision on the analysis that continues, etc.

The linear model of Koiter (and its variants, see [5] and [6]) is one of the most used since it couples the membrane and flexion effects; the book [1] is an excellent summary of conditions for the existence and uniqueness of a solution of the model, approximation through finite element methods, etc. In this work we develop the previous step of analyzing the origin of the different variants of the model, to justify the

${ }^{1}$ This work is part of the Human Capital and Mobility Program "Shells: Mathematical Modeling and Analysis, Scientific Computing" of the Commission of the European Communities (Contract ERBCHRXCT940536). 
equations that appear and study the error order of the differences among them.

To visualize better the differences among the different versions and how they are sensitive to the variation of certain parameters (for example, the thickness of the shell), we have approximated the two more usual variants of the model, using Sander's "delinquent" nonconforming finite element method, whose implementation has permitted us to numerically compare the results.

Throughout the work we use the classic notation of differential geometry of surfaces and volumes (in particular, the summation convention for a Greek or Latin repeated index, and the notation $v_{\alpha \mid \beta}$ or $V_{i \| j}$ for covariant derivatives).

\section{Deformation of a shell}

Given $\Omega \subset \mathbb{R}^{2}$ a bounded domain, with a Lipschitz-continuous boundary $\Gamma$, the middle surface $\overline{\mathcal{S}}$ of a shell $\overline{\mathcal{C}}$ is defined as the image of $\bar{\Omega}$ by mapping $\vec{\phi}$; we assume that $\vec{\phi}$ is of class $\mathcal{C}^{3}\left(\bar{\Omega} ; \mathbb{R}^{3}\right)$ and that the vectors $\vec{a}_{\alpha}=\vec{\phi}_{, \alpha}$ are linearly independent. We denote by $\left(a_{\alpha \beta}\right)$ and $\left(b_{\alpha \beta}\right)$ the covariant components of the first and second fundamental form of the surface $\overline{\mathcal{S}}$ and let $a=\operatorname{det}\left(a_{\alpha \beta}\right)$. Also, $\left\{\vec{a}_{\alpha}\right\}$ and $\left\{\vec{a}^{\alpha}\right\}$ are the covariant and contravariant basis of the tangent plane for all points of $\overline{\mathcal{S}}$ and let $\vec{a}_{3}=\left(\vec{a}_{1} \times \vec{a}_{2}\right) / \sqrt{a}$ be the normal and unitary vector.

A shell is defined then as the closed subset of $\mathbb{R}^{3}$

$$
\overline{\mathcal{C}}=\left\{M \in \mathbb{R}^{3}: \overrightarrow{O M}=\vec{\phi}(\xi)+\xi^{3} \vec{a}_{3}(\xi) ; \xi \in \bar{\Omega} ; \frac{-e(\xi)}{2} \leq \xi^{3} \leq \frac{e(\xi)}{2}\right\}
$$

$e$ being a function of class $\mathcal{C}^{0}\left(\bar{\Omega} ; \mathbb{R}_{+}\right)$such that it describes the thickness. We make a first thinness hypothesis in the sense that in each point:

$$
e \leq \frac{1}{\aleph} \min \left\{\left|R_{N}\right|\right\} \quad\left(R_{N} \text { normal curvature radio }\right)
$$

where $\aleph$ is a "large enough" number; furthermore, evidently, $e$ must be "small" in comparison with the other two dimensions of the shell, whose orders of magnitude we denote by $L$. Also, the shell $\overline{\mathcal{C}}$ is obtained by:

$$
\vec{\Phi}: \overline{\Omega^{e}} \rightarrow \mathbb{R}^{3}, \quad \overline{\Omega^{e}}=\bar{\Omega} \times\left[-\frac{e}{2}, \frac{e}{2}\right], \quad \vec{\Phi}=\vec{\phi}+\xi^{3} \vec{a}_{3}
$$

we denote by $\left\{\vec{g}_{i}\right\}$ and $\left\{\vec{g}^{i}\right\}$ the covariant and contravariant basis of the tangent space, and $\left(g_{i j}\right)$ and $\left(g^{i j}\right)$ are the components of the metric tensor. 
We consider the boundary problem that describes the equilibrium of the shell $\overline{\mathcal{C}}$, submitted to the action of different forces and clamped on a part of its lateral boundary; let $\overline{\mathcal{C}}^{*}$ be the deformed configuration and we denote by $\vec{U}: \overline{\mathcal{C}} \rightarrow \overline{\mathcal{C}}^{*}$ the corresponding displacement field, $\vec{u}$ being its restriction to $\overline{\mathcal{S}}$. We see below how, under the acceptance of different hypothesis, $\vec{U}$ is determined as function of $\vec{u}$, that, at the same time, is decomposed in the form $\vec{u}=u_{i} \vec{a}^{i}$; the utilization of the map $\vec{\phi}$ permits the conclusion that the effective unknowns of the problem are, thus, the functions $u_{i}: \bar{\Omega} \rightarrow \mathbb{R}(i=1,2,3)$.

In addition to accepting the previous thinness hypothesis, we work on a linear theory: small loads that imply small displacement; this hypothesis permits the simplification of expressions in which appear powers of $u_{i}$ of different orders. The following is obtained:

Proposition 1. Denoting with a bar above the concepts referring to the deformed middle surface, it is verified that:

(4) $\bar{a}_{\alpha \beta}-a_{\alpha \beta}=u_{\alpha \mid \beta}+u_{\beta \mid \alpha}-2 b_{\alpha \beta} u_{3}$,

$$
\begin{aligned}
\bar{b}_{\alpha \beta}-b_{\alpha \beta}= & -0.5\left(\theta_{\alpha \mid \beta}(\vec{u})+\theta_{\beta \mid \alpha}(\vec{u})-b_{\alpha}^{\lambda} u_{\lambda \mid \beta}-b_{\beta}^{\lambda} u_{\lambda \mid \alpha}\right) \\
& -b_{\alpha}^{\lambda} b_{\lambda \beta} u_{3} .
\end{aligned}
$$

Proof: First, for the deformed middle surface we have:

$$
\overrightarrow{\bar{\phi}}=\vec{\phi}+\vec{u}, \quad \overrightarrow{\vec{a}}_{\alpha}=\vec{a}_{\alpha}+\vec{u}_{, \alpha}
$$

with the aid of the Gauss and Weingarten formulae we have

$$
\vec{u}_{, \alpha}=\left(u_{\beta \mid \alpha}-b_{\beta \alpha} u_{3}\right) \vec{a}^{\beta}-\theta_{\alpha}(\vec{u}) \vec{a}_{3} .
$$

Finally, by remembering the concepts of the normal vector and the fundamental forms, and by repeatedly applying the linear assumption we obtain the result.

We recall that $\vec{\theta}(\vec{u})$ is known as the vector of rotation of the normal and that the covariant components of the strain and change of curvature tensors of the middle surface $\overline{\mathcal{S}}$ are respectively defined by:

$$
\gamma_{\alpha \beta}(\vec{u})=0.5\left(\bar{a}_{\alpha \beta}-a_{\alpha \beta}\right), \quad \bar{\varrho}_{\alpha \beta}(\vec{u})=\bar{b}_{\alpha \beta}-b_{\alpha \beta} .
$$




\section{The Kirchhoff-Love assumptions}

Most authors derive their equations on the basis of the "classic" Kirchhoff-Love assumptions:

H1 The normal to the undeformed middle surface, considered as a set of points, remains normal to the deformed middle surface, and the displacements in the direction of the normals are independent of the position on the middle surface.

$\mathrm{H} 2$ The effect of the normal stress, on surfaces parallel to the middle surface, may be neglected in the stress-strain relations.

These assumptions have an approximate character; nevertheless, they are contradictory as we prove now. For it, we consider a kinematically admissible displacement field $\vec{V}$, the linear strain tensor $\varepsilon(\vec{V})$ defined by

$$
\varepsilon(\vec{V})=\frac{1}{2}(\nabla \vec{V}+\overrightarrow{\nabla \vec{V}})=\varepsilon_{i j}(\vec{V}) \vec{g}^{i} \otimes \vec{g}^{j},
$$

and the Piola-Kirchhoff stress tensor $\sigma(\vec{V})$ defined by

$$
\sigma(\vec{V})=\frac{E \nu}{(1+\nu)(1-2 \nu)} \operatorname{tr}(\varepsilon(\vec{V})) I+\frac{E}{1+\nu} \varepsilon(\vec{V})=\sigma^{i j}(\vec{V}) \vec{g}_{i} \otimes \vec{g}_{j}
$$

(for an elastic, homogeneous and isotropic material, of Young's modulus $E$ and Poisson's coefficient $\nu$ ), and we operate with them.

Proposition 2. As consequence of $H 1$ it is deduced that

$$
\vec{V}=\vec{v}+d\left(\xi^{3}\right) \vec{\theta}(\vec{v})+\left(d\left(\xi^{3}\right)-\xi^{3}\right) \vec{a}_{3},
$$

where $d$ is the function that measures the elongations on the normal. Furthermore:

$$
\begin{aligned}
\stackrel{\circ}{\varepsilon}_{\alpha \beta}(\vec{V}) & =\gamma_{\alpha \beta}(\vec{v}), \\
\stackrel{\circ}{\varepsilon}_{\alpha \beta, 3}(\vec{V}) & =-\bar{\varrho}_{\alpha \beta}(\vec{v}), \\
\stackrel{\circ}{\varepsilon}_{\alpha \beta \| 3}(\vec{V}) & =-\bar{\varrho}_{\alpha \beta}(\vec{v})+b_{\alpha}^{\lambda} \gamma_{\lambda \beta}(\vec{v})+b_{\beta}^{\lambda} \gamma_{\alpha \lambda}(\vec{v}), \\
\stackrel{\circ}{\varepsilon}_{\alpha 3}(\vec{V}) & =\stackrel{\circ}{\varepsilon}_{33}(\vec{V})=\stackrel{\circ}{\varepsilon}_{\alpha 3,3}(\vec{V})=\stackrel{\circ}{\varepsilon}_{33,3}(\vec{V})=\stackrel{\circ}{\varepsilon}_{\alpha 3 \| 3}(\vec{V})=\stackrel{\circ}{\varepsilon}_{33 \| 3}(\vec{V})=0,
\end{aligned}
$$

where we denote the values at the middle surface with a small circle above functions.

Proof: With the mathematical expressions of the Assumption H1, the Relation (6) is a simple consequence of (3). From here, we differentiate 
this expression following the definition of $\varepsilon(\vec{V})$ and by evaluating at the middle surface we obtain the relations for $\stackrel{\circ}{\varepsilon}_{i j}(\vec{V})$ and for their derivatives; we remark that to obtain the final expressions we use:

$$
d(0)=0, \quad d^{\prime}(0)=1, \quad d^{\prime \prime}(0)=0
$$

since the function $d$ has in the proximity of the middle surface an analogous behavior to the identity (see [9] for the details).

Proposition 3. As consequence of H2 it is deduced that

$$
\varepsilon_{33}(\vec{V})=-\frac{\nu}{1-\nu} g^{\alpha \beta} \varepsilon_{\alpha \beta}(\vec{V})
$$

Proof: The Assumption $\mathrm{H} 2$ is equivalent to asserting that $\sigma^{33}(\vec{V})$ is zero; on the other hand, the definition of $\sigma(\vec{V})$ permits us to write:

$\sigma^{i j}(\vec{V})=\mathcal{E}^{i j k l} \varepsilon_{k l}(\vec{V})$, with $\mathcal{E}^{i j k l}=\frac{E \nu g^{i j} g^{k l}}{(1+\nu)(1-2 \nu)}+\frac{E\left(g^{i k} g^{j l}+g^{i l} g^{j k}\right)}{2(1+\nu)}$

by combining it with the expressions of the components $\left(g^{i j}\right)$ (deduced from (2)) we obtain (7).

We have already sufficient elements to show the contradiction of the classic Kirchhoff-Love assumptions. In fact, by evaluating (7) at the middle surface and taking into account the results in Proposition 2 we arrive at the fact that the trace of the strain tensor of the middle surface $\left(\gamma_{\alpha \beta}\right)$ is zero, and it is not necessarily true for an arbitrary shell.

In [5], Koiter introduces a new "modified" version of the KirchhoffLove assumptions, substituting $\mathrm{H} 1$ for:

H1' The effect of the transverse shear stresses, acting on surfaces parallel to the middle surface, may be neglected in the stress-strain relations.

Let see its consistency and the modifications that it introduces (compare with the results of Proposition 2).

Proposition 4. As consequence of $H 1^{\prime}$ it is deduced that:

$$
\begin{aligned}
\stackrel{\circ}{\varepsilon}_{\alpha \beta}(\vec{V}) & =\gamma_{\alpha \beta}(\vec{v}), \\
\stackrel{\circ}{\varepsilon}_{\alpha \beta \| 3}(\vec{V}) & =-\bar{\varrho}_{\alpha \beta}(\vec{v})+b_{\alpha}^{\lambda} \gamma_{\lambda \beta}(\vec{v})+b_{\beta}^{\lambda} \gamma_{\alpha \lambda}(\vec{v})+\frac{\nu}{1-\nu} b_{\alpha \beta} a^{\lambda \mu} \gamma_{\lambda \mu}(\vec{v}), \\
\stackrel{\circ}{\varepsilon}_{\alpha 3}(\vec{V}) & =\stackrel{\circ}{\varepsilon}_{\alpha 3 \| 3}(\vec{V})=0
\end{aligned}
$$


furthermore, for the displacement field $\vec{V}$ we have

$$
\vec{V}=\vec{v}+\xi^{3} \vec{\theta}(\vec{v})+\xi^{3} \stackrel{\circ}{\varepsilon}_{33}(\vec{V}) \vec{a}^{3} .
$$

Proof: The Assumption H1' is equivalent to stating that $\sigma^{\alpha 3}(\vec{V})$ is zero; by working as in Proposition 3 we arrive at the fact that $\varepsilon_{\alpha 3}(\vec{V})$ is also zero. From here the expressions of $\stackrel{\circ}{\varepsilon}_{i j}(\vec{V})$ and their derivatives are deduced from the customary rules of the covariant derivation. Making a Taylor's limited development of first order (linear theory) in each point of the middle surface, in the form

$$
\vec{V}=\stackrel{\circ}{\vec{V}}+\xi^{3} \stackrel{\circ}{\vec{V}}, 3_{,}
$$

it is easy to see that:

$$
\stackrel{\circ}{\vec{V}}=\vec{v} \quad \text { and } \quad \stackrel{\circ}{V}_{, 3}=\vec{\theta}(\vec{v})+\stackrel{\circ}{\varepsilon} 33(\vec{V}) \vec{a}_{3},
$$

and from here we arrive finally at (8).

It is important to note that the Assumptions H1' and $\mathrm{H} 2$ do not present contradiction in their formulation, since there is not an ambiguity for $\stackrel{\circ}{\varepsilon}_{33}(\vec{V})$, for which the expression (7) is conserved, and

$$
\varepsilon_{33 \| 3}(\vec{V})=-\frac{\nu}{1-\nu} g^{\alpha \beta} \varepsilon_{\alpha \beta \| 3}(\vec{V})
$$

for its derivative. Also, we indicate that (8) permits the possibility of small shears on the normal component of the displacement.

In the following, we will suppose that the shell is fixed on a part $C_{0}$ of its lateral boundary. A simple calculation from (8) permits us to conclude that:

$$
\vec{v}=\overrightarrow{0}, \quad v_{3, \nu}=0, \text { on } S_{0},
$$

where $S_{0}$ is the projection on $\overline{\mathcal{S}}$ of $C_{0}$ and $\vec{\nu}$ is the outward unit normal vector to $S_{0}$; this is known as clamped conditions on $S_{0}$.

\section{Koiter's linear model}

The basic idea of Koiter's shell theory is to effect an integration on the thickness; we recall that the strain energy of a solid for an admissible displacement field $\vec{V}$, is given by

$$
\mathcal{A}(\vec{V})=\frac{1}{2} \int_{\mathcal{C}} \operatorname{tr}(\sigma(\vec{V}) \circ \varepsilon(\vec{V})) d M=\frac{1}{2} \int_{\mathcal{C}} \sigma^{i j}(\vec{V}) \varepsilon_{i j}(\vec{V}) d M .
$$


Proposition 5. Under the acceptance of the thinness hypothesis, the strain energy of the shell admits the expression

(11)

$$
\mathcal{A}(\vec{V})=\frac{1}{2} \int_{\mathcal{S}} e E^{\alpha \beta \lambda \mu}\left(\stackrel{\circ}{\varepsilon}_{\alpha \beta}(\vec{V}) \stackrel{\circ}{\varepsilon}_{\lambda \mu}(\vec{V})+\frac{e^{2}}{12} \stackrel{\circ}{\varepsilon}_{\alpha \beta \| 3}(\vec{V}) \stackrel{\circ}{\varepsilon}_{\lambda \mu \| 3}(\vec{V})\right) d P,
$$

where the tensor of elastic moduli $\left(E^{\alpha \beta \lambda \mu}\right)$ is given by

$$
E^{\alpha \beta \lambda \mu}=\frac{E}{2(1+\nu)}\left(a^{\alpha \lambda} a^{\beta \mu}+a^{\alpha \mu} a^{\beta \lambda}+\frac{2 \nu}{1-\nu} a^{\alpha \beta} a^{\lambda \mu}\right) .
$$

Proof: We operate in the integrand using Taylor's limited development in each point of the middle surface in the form

$$
\Psi(\vec{V})=\sigma^{i j}(\vec{V}) \varepsilon_{i j}(\vec{V})=\stackrel{\circ}{\Psi}(\vec{V})+\xi^{3} \stackrel{\circ}{\Psi}_{\| 3}(\vec{V})+\frac{1}{2}\left(\xi^{3}\right)^{2} \stackrel{\circ}{\Psi}_{\| 33}(\vec{V}) .
$$

Developing this relation, effecting the integration on the thickness (we note that $\sqrt{g}$ and $\sqrt{a}$ are the volume and surface elements, respectively) and simplifying to the maximum, we obtain

$$
\int_{-e / 2}^{e / 2} \Psi(\vec{V}) \frac{\sqrt{g}}{\sqrt{a}} d \xi^{3}=e \stackrel{\circ}{\mathcal{E}}^{i j k l}\left(\stackrel{\circ}{\varepsilon}_{i j}(\vec{V}) \stackrel{\circ}{\varepsilon}_{k l}(\vec{V})+\frac{e^{2}}{12} \stackrel{\circ}{\varepsilon}_{i j \| 3}(\vec{V}) \stackrel{\circ}{\varepsilon}_{k l \| 3}(\vec{V})\right) ;
$$

from here we deduce (11) making the substitutions (7) and (9). It is important to indicate that in the previous simplification we have neglected all those terms whose order of magnitude is $O(1 / \aleph), O\left(1 / \aleph^{2}\right)$ or $O\left(e^{2} / L^{2}\right)$, with respect to some of the conserved terms; as we can see, the accomplished thinness hypothesis causes this simplification to be admissible, because these terms are "indeed negligible".

It remains only to substitute the components $\stackrel{\circ}{\varepsilon}_{\alpha \beta}(\vec{V})$ and their covariant derivatives by their corresponding expressions deduced as a consequence of the Kirchhoff-Love assumptions; with respect to the first there is no problem, but for the second we see that neither of the previously obtained expressions permits us to arrive at Koiter's classic model, which is directly obtained substituting in (11) the components $\stackrel{\circ}{\varepsilon \beta}_{\alpha \| 3}(\vec{V})$ by $-\bar{\varrho}_{\alpha \beta}(\vec{v})$. It can be observed that with the Asssumption H1 we obtain the previous relation by differentiating $\varepsilon_{\alpha \beta}(\vec{V})$ in the usual way, and not in the covariant way.

In [6] Koiter introduces a modified model by making the following substitution for the derivatives of the linear strain tensor

$$
\stackrel{\circ}{\varepsilon}_{\alpha \beta \| 3}(\vec{V})=-\bar{\varrho}_{\alpha \beta}(\vec{v})+\frac{1}{2} b_{\alpha}^{\lambda} \gamma_{\lambda \beta}(\vec{v})+\frac{1}{2} b_{\beta}^{\lambda} \gamma_{\alpha \lambda}(\vec{v}) ;
$$


this is, in a certain way, an arithmetic mean among the expression used in the classic model and the relation obtained as a consequence of the Kirchhoff-Love assumptions.

Nevertheless, we can see that the addition to $\stackrel{\circ}{\varepsilon}_{\alpha \beta \| 3}(\vec{V})$ of terms of the form $C b_{\alpha}^{\lambda} \gamma_{\lambda \beta}(\vec{v})(C$ small constant) produces in (11) addends whose order of magnitude is $O(1 / \aleph)$ or $O\left(1 / \aleph^{2}\right)$ with respect to the already existing (the same proof of Proposition (5) is valid); again, by recalling the thinness hypothesis, the variety of the existing formulations is justified.

Before ending this section we remark that with the potential energy of exterior forces $\mathcal{F}(\vec{V})$ we can accomplish the same limited development of Taylor and the subsequent process of simplification and integration on the thickness; then, we obtain

$$
\mathcal{F}(\vec{V})=\int_{\mathcal{S}} p^{i} v_{i} d M+\int_{S_{1}}\left(N^{i} v_{i}+M^{\alpha} \theta_{\alpha}(\vec{v})\right) d \sigma,
$$

where $\vec{p}=p^{i} \vec{a}_{i}$ is the superficial density on $\overline{\mathcal{S}}$ of the volume loads applied and $\vec{N}=N^{i} \vec{a}_{i}$ and $\vec{M}=\varepsilon_{\alpha \beta} M^{\beta} \vec{a}^{\alpha}$ are, respectively, the linear density and the resulting linear moment on $S_{1}$ (the part of the boundary of $\overline{\mathcal{S}}$ complementary to $S_{0}$ ) of the boundary loads applied.

\section{Variational formulation of the problem}

The variational formulation of the problem and the establishment of the existence and uniqueness of a solution requires a previous step that is the formulation on the reference domain $\bar{\Omega}$, which is made through a simple variable change in the corresponding integral.

Taking into account the expressions of the strain and the change of curvature tensors of the middle surface and adding the boundary conditions, we arrive at the space of admissible displacement

$$
\overrightarrow{\mathbf{V}}=\left\{\vec{v}=\left(v_{1}, v_{2}, v_{3}\right) \in\left(H^{1}(\Omega)\right)^{2} \times H^{2}(\Omega): v_{i}=v_{3, n}=0 \text { on } \Gamma_{0}\right\},
$$

equipped with the Sobolev usual norm. It can be proved that the strain energy $\mathcal{A}$ and the potential energy of exterior forces $\mathcal{F}$ are associated, respectively, to a symmetric bilinear form $a$ and to a linear form $f$, defined on $\overrightarrow{\mathbf{V}}$ and both $\overrightarrow{\mathbf{V}}$-continuous; their expressions are:

$$
\begin{aligned}
a(\vec{u}, \vec{v}) & =\int_{\Omega} e E^{\alpha \beta \lambda \mu}\left(\gamma_{\alpha \beta}(\vec{v}) \gamma_{\lambda \mu}(\vec{u})+\frac{e^{2}}{12} \bar{\varrho}_{\alpha \beta}(\vec{v}) \bar{\varrho}_{\lambda \mu}(\vec{u})\right) \sqrt{a} d \xi \\
f(\vec{v}) & =\int_{\Omega} p^{i} v_{i} \sqrt{a} d \xi+\int_{\Gamma_{1}}\left(N^{i} v_{i}+M^{\alpha} \theta_{\alpha}(\vec{v})\right)\left|\vec{\phi}_{, t}\right| d \gamma
\end{aligned}
$$

So, the principle of virtual work allows us to write the variational formulation of the problem, i.e.:

Problem 1. Find $\vec{u} \in \overrightarrow{\mathbf{V}}$ such that $a(\vec{u}, \vec{v})=f(\vec{v})$, for all $\vec{v} \in \overrightarrow{\mathbf{V}}$. 
Theorem 1. Problem 1 has one and only one solution.

We refer to [1] (and to the references given there) for the study of the existence and uniqueness of the solution of Problem 1; nevertheless, we indicate that the proof given there can be generalized to any variant of Koiter's linear model since the theorem of the rigid body in which it is based is also valid under expressions of the type (12).

\section{Approximation by F.E.M.}

We approximate the shell problem using Sander's "delinquent" nonconforming finite element method (see [8]), which uses the $P_{2}$-Lagrangeedge triangle for the approximation of the tangential components of the displacement, and Sander's delinquent triangle for the normal component. The definition of these two finite elements is described in Figure 1, using the classic notation of [4].

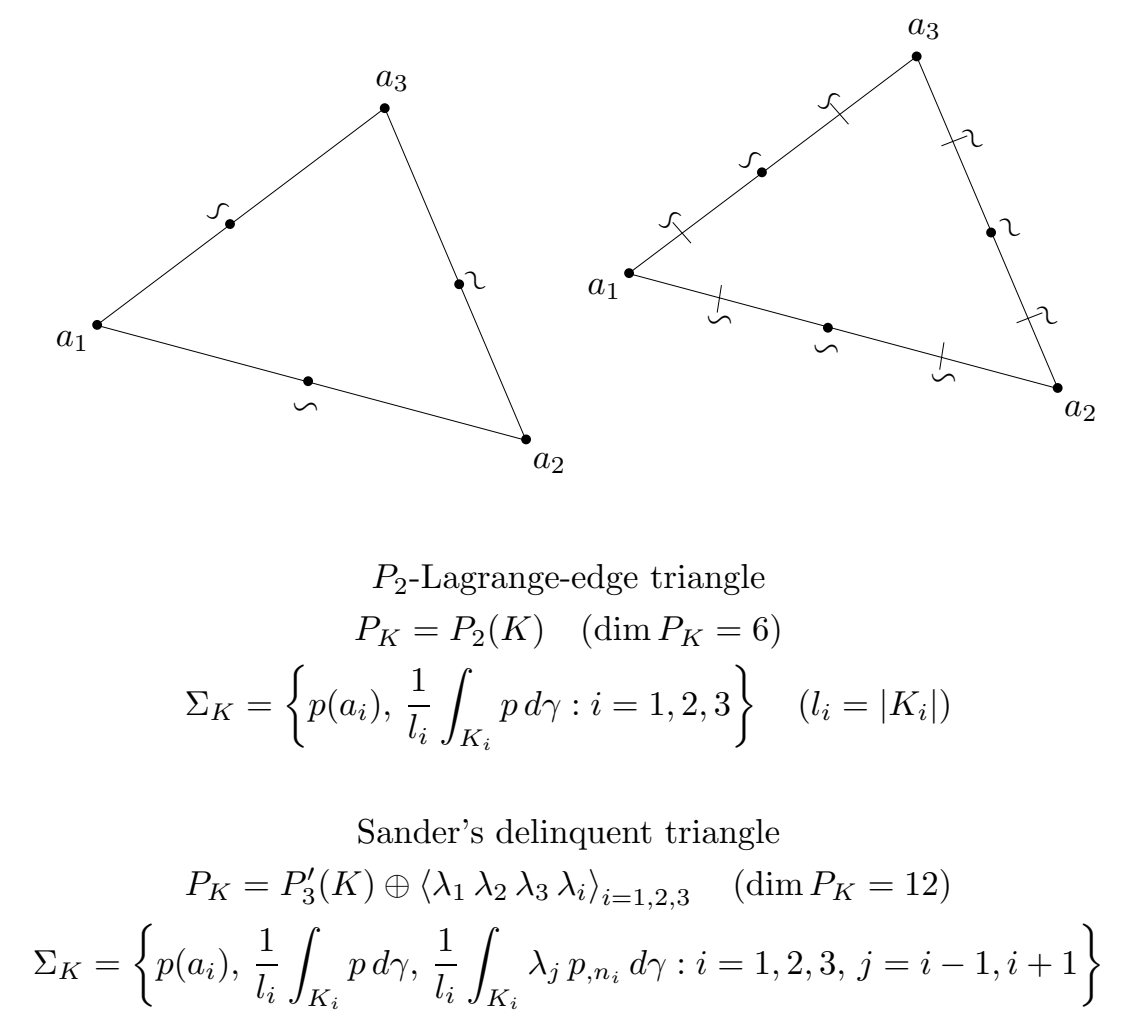

Figure 1. Sander's "delinquent" finite element method 
We indicate that it is a nonconforming method, since the second finite element is not of class $\mathcal{C}^{1}$ (and neither is $\mathcal{C}^{0}$ ); so, if we build the finite element spaces $\overrightarrow{\mathbf{V}}_{h}$ associated to a regular family of triangulations $\mathcal{T}_{h}$ of $\bar{\Omega}$ in the usual way, we have:

$$
\overrightarrow{\mathbf{V}}_{h} \subset\left(H^{1}(\Omega)\right)^{2} \times \prod_{K \in \mathcal{T}_{h}} H^{2}(K) \subset\left(H^{1}(\Omega)\right)^{2} \times L^{2}(\Omega), \quad \overrightarrow{\mathbf{V}}_{h} \not \subset \overrightarrow{\mathbf{V}} ;
$$

also, the bilinear form $a$ and the linear form $f$ have to be approximated in $\overrightarrow{\mathbf{V}}_{h}$ by:

$$
\begin{aligned}
a_{h}\left(\vec{u}_{h}, \vec{v}_{h}\right)= & \int_{\Omega} e E^{\alpha \beta \lambda \mu} \gamma_{\alpha \beta}\left(\vec{v}_{h}\right) \gamma_{\lambda \mu}\left(\vec{u}_{h}\right) \sqrt{a} d x d y \\
& +\sum_{K \in \mathcal{T}_{h}} \int_{K} \frac{e^{3}}{12} E^{\alpha \beta \lambda \mu} \bar{\varrho}_{\alpha \beta}\left(\vec{v}_{h}\right) \bar{\varrho}_{\lambda \mu}\left(\vec{u}_{h}\right) \sqrt{a} d x d y, \\
f_{h}\left(\vec{v}_{h}\right)= & \int_{\Omega} p^{i} v_{h i} \sqrt{a} d x d y+\sum_{K \in \mathcal{T}_{h}} \int_{K \cap \Gamma_{1}}\left(N^{i} v_{h i}+M^{\alpha} \theta_{\alpha}\left(\vec{v}_{h}\right)\right)\left|\vec{\phi}_{, t}\right| d \gamma .
\end{aligned}
$$

We can already to state the discrete problem:

Problem 2. Find $\vec{u}_{h} \in \overrightarrow{\mathbf{V}}_{h}$ such that $a_{h}\left(\vec{u}_{h}, \vec{v}_{h}\right)=f_{h}\left(\vec{v}_{h}\right)$, for all $\vec{v}_{h} \in \overrightarrow{\mathbf{V}}_{h}$.

The analysis, the resolution and the implementation of this problem has been realized in $[\mathbf{3}],[\mathbf{9}]$ and $[\mathbf{7}]$, and the following is obtained:

Theorem 2. Problem 2 admits a unique solution satisfying

$$
\left\|\vec{u}-\vec{u}_{h}\right\|_{h}=O\left(h^{2}\right)
$$

if $\vec{u}_{h} \in\left(H^{3}(\Omega)\right)^{2} \times H^{4}(\Omega)$, where $\|\cdot\|_{h}$ denotes the Sobolev usual norm in the space $\left(H^{1}(\Omega)\right)^{2} \times \prod_{K \in \mathcal{T}_{h}} H^{2}(K)$.

\section{Numerical tests}

In order to illustrate the previous considerations, we present some numerical experiments derived using Sander's finite element method on the two versions of Koiter's linear model. These tests have been obtained with the aid of the MODULEF Library (see [2]), in which we have implemented the previous methods.

The first experiment is a hyperbolic paraboloid which is clamped and submitted to a uniform pressure of density $q$; a sketching of this shell as well as the physical data of the discussed problem are given in Figure 2. 


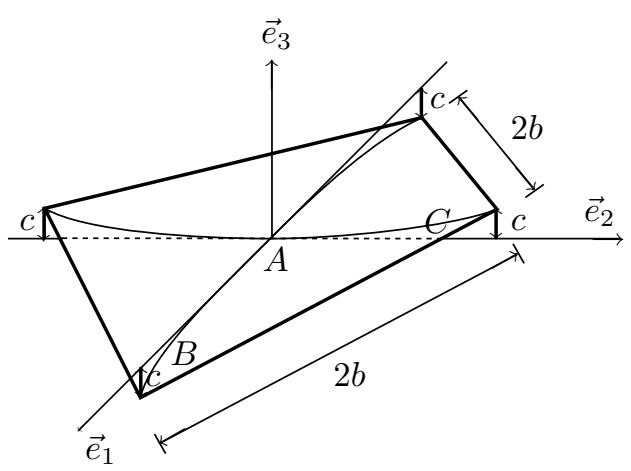

$$
\begin{aligned}
E & =2.85 * 10^{4} \\
\nu & =0.4 \\
e & =0.8 \\
q & =0.01 \\
b & =50 . \\
c & =10 .
\end{aligned}
$$

Figure 2. Hyperbolic paraboloid

In Figure 3 we show the deformations along the sides $A B$ and $A C$. With the reference configuration (the nodes of the triangulation are labeled with $*$ ), we also show the deformed configurations (labeled with o). We note that in these figures, whose deformations are magnified with a coefficient of 200 for better visualization, we exhibit the results obtained with the classic model and with the modified model, but its graphs are superimposed (even with the above-mentioned magnification) because of the similar behaviour of the numerical results.
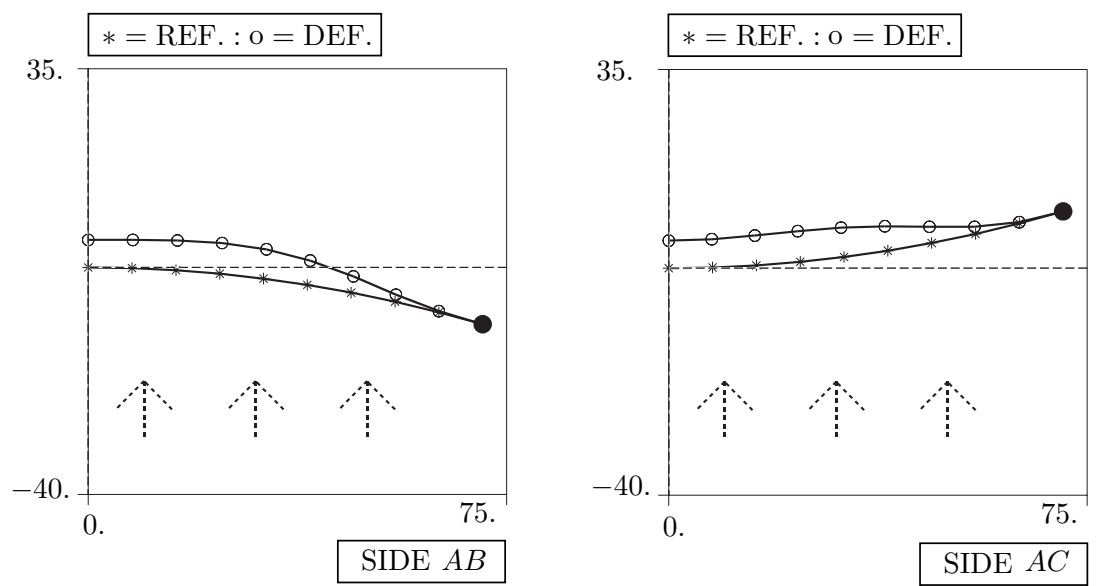

Figure 3. Hyperbolic paraboloid: deformations 


\begin{tabular}{|c|c|c|c|r|}
\hline Thickness & $U_{3}(A)$ clas. & $U_{3}(A)$ modif. & \% Relat. error & \% Change \\
\hline$e / 3$ & 0.0416920940 & 0.0416888700 & 0.0077326890 & 0.480 \\
\hline$e / 2$ & 0.0297627322 & 0.0297600199 & 0.0091130410 & 0.566 \\
\hline$e$ & 0.0242857928 & 0.0242897008 & 0.0160917538 & 1.000 \\
\hline $2 e$ & 0.0152566484 & 0.0152673617 & 0.0702202060 & 4.363 \\
\hline $3 e$ & 0.0092417958 & 0.0092529293 & 0.1204689351 & 7.486 \\
\hline $4 e$ & 0.0057903262 & 0.0057998190 & 0.1639437514 & 10.188 \\
\hline $5 e$ & 0.0037729762 & 0.0037804555 & 0.1982337968 & 12.318 \\
\hline
\end{tabular}

Table 1. Hyperbolic paraboloid: results

In Table 1 we give the results obtained in the center of the paraboloid (point $A$ ) when we change the thickness of the shell; in this way, taking the value $e=0.8$ as a reference, we have analyzed the results for $e / 3$, $e / 2,2 e, 3 e, 4 e$ and $5 e$. As can be observed, the percentage of change is sensitive to the increase in the thickness, something that was expected, since the differences between these models are terms of the order $O(1 / \aleph)$ or $O\left(1 / \aleph^{2}\right)$, and $\aleph$ is related with $e$ through $(1)$.

The second experiment is the classic test carried out on a cylindrical roof with a rigid diaphragm fixed to its two curved sides that only permits movements in the direction of the axis of the cylinder, and submitted to its own weight of load density $q$. In Figure 4 we give the data and the characteristics of the problem.

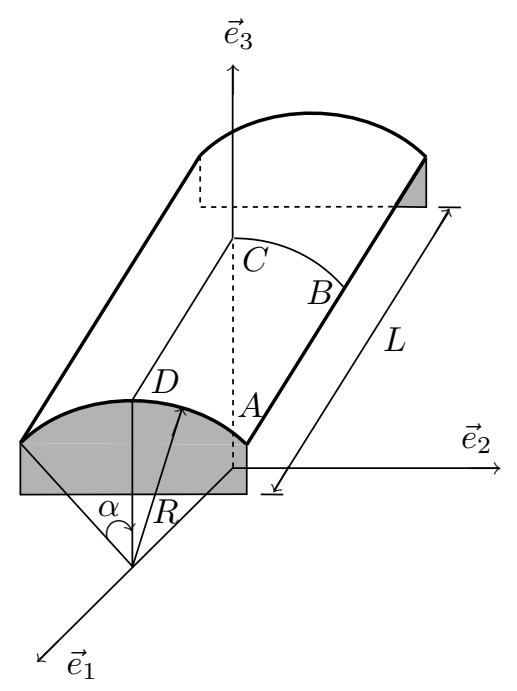

$$
\begin{aligned}
E & =3 . * 10^{6} \\
\nu & =0 . \\
e & =3 . \\
q & =-0.625 \\
R & =300 . \\
L & =600 . \\
\alpha & =40^{\circ} .
\end{aligned}
$$

Figure 4. Cylindrical roof 


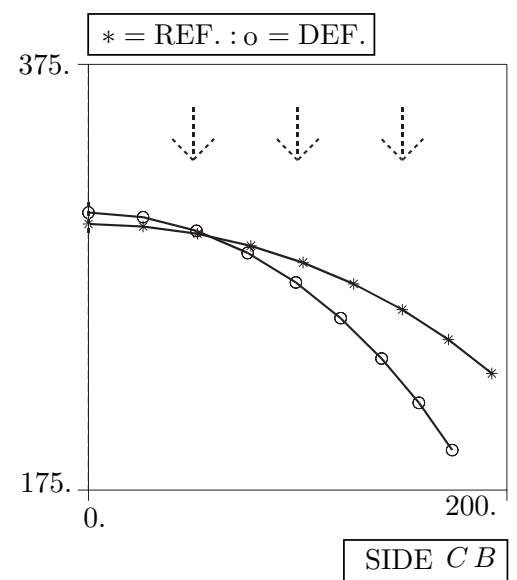

Figure 5. Cylindrical roof: deformations

We consider the central half-arch $B C$, and we give in Figure 5 the reference configuration (labeled with $*$ ) and the deformed configurations (labeled with o) corresponding to the classic model and to the modified model. As in the previous example, for better visualization, the deformations are amplified with a coefficient 100; in spite of this, the graphs corresponding to the two models are superimposed.

We conclude carrying out the same study of the sensitivity of the model change with respect to the thickness change, by examining the vertical displacement of the point $B$ (in absolute value, the greater displacement of the shell). As can be noted, the results are totally analogous for the behavior of the percentages of change (we indicate that in this case the lower values for the percentages of change are due to the existence of a unique curvature in the structures of cylindrical type).

\begin{tabular}{|c|c|c|c|r|}
\hline Thickness & $U_{3}(B)$ clas. & $U_{3}(B)$ modif. & \% Relat. error & \% Change \\
\hline$e / 3$ & -34.79117673 & -34.79133249 & 0.000447700 & 0.160 \\
\hline$e / 2$ & -16.19772821 & -16.19787827 & 0.000926426 & 0.331 \\
\hline$e$ & -3.59804675 & -3.59814735 & 0.002795878 & 1.000 \\
\hline $2 e$ & -0.66375276 & -0.66380562 & 0.007965059 & 2.848 \\
\hline $3 e$ & -0.26131452 & -0.26134978 & 0.013491672 & 4.825 \\
\hline $4 e$ & -0.14486851 & -0.14489491 & 0.018224940 & 6.518 \\
\hline $5 e$ & -0.09595338 & -0.09597447 & 0.021983488 & 7.862 \\
\hline
\end{tabular}

Table 2. Cylindrical roof: results 


\title{
References
}

1. M. Bernadou, "Méthode d'éléments finis pour les problèmes de coques minces," Masson, Paris, 1994.

2. M. Bernadou, P. L. George, A. Hassim, P. Joly, P. Laug, B. Muller, A. Perronnet, E. Saltel, D. Steer, G. VanderBORK AND M. VIDRASCU, "MODULEF: une bibliothèque modulaire d'éléments finis," Editions INRIA, Rocquencourt, 1988.

3. M. Bernadou And P. Trouvé, The delinquent finite element method for the analysis of thin branched shells (to appear).

4. P. G. CiARlet, "The Finite Element Method for Elliptic Problems," North-Holland, Amsterdam, 1978.

5. W. T. KoIter, A consistent first approximation in the general theory of thin elastic shells, Proc. I.U.T.A.M., Symposium on the Theory of Thin Elastic Shells (1960), 12-33.

6. W. T. Koiter, On the foundations of the linear theory of thin elastic shells, Proc. Kon. Ned. Ak. Wet. B73 (1970), 169-195.

7. F. PAlma AND A. Vilariño, Approximation des problèmes généraux de coques dans des espaces d'éléments finis non conformes, Rapport de Recherche INRIA 2603 (1995).

8. G. SANDER, Modèles d'éléments finis délinquants, Groupe de travail méthodes numériques pour les problèmes de coques, Université de Liége, 1985.

9. A. Vilariño, Contribución al estudio del modelo lineal de láminas delgadas de W. T. Koiter: Fundamentación matemática y aproximación mediante el método no conforme de elementos finitos delincuente de Sander, Tesis Doctoral, Universidad de Málaga, 1994.

\author{
Departamento de Análisis Matemático \\ Facultad de Ciencias \\ Universidad de Málaga \\ Campus de Teatinos \\ 29080 Málaga \\ SPAIN
}

Rebut el 23 d'Octubre de 1995 